\title{
New in situ solid-state NMR strategies for exploring materials formation and adsorption processes: prospects in heterogenous catalysis
}

\author{
Kenneth D. M. Harris ${ }^{1}$
}

Received: 2 February 2016/Accepted: 21 April 2016/Published online: 20 May 2016

(c) The Author(s) 2016. This article is published with open access at Springerlink.com

\begin{abstract}
Solid-state NMR spectroscopy is a powerful technique for studying structural and dynamic properties of solids and has considerable potential to be exploited for in situ studies of chemical processes. However, adapting solid-state NMR techniques and instrumentation for in situ applications are often associated with technical challenges, and for this reason, the opportunities remain underexploited. This paper highlights two experimental strategies that we have developed in recent years for in situ solid-state NMR investigations of solidstate processes. One technique is focused on probing details of the time evolution of materials formation processes, and the other technique is focused on understanding the time evolution of adsorption processes in microporous and mesoporous solid host materials. Each of these in situ solid-state NMR techniques has significant prospects for applications in areas relating to heterogeneous catalysis.
\end{abstract}

Keywords Solid-state NMR - In situ techniques · Adsorption processes - Materials formation processes . Crystallization

\section{Introduction}

It is well recognized that the development and application of experimental techniques for in situ investigations of chemical processes, such as heterogeneous

Kenneth D. M. Harris

HarrisKDM@cardiff.ac.uk

1 School of Chemistry, Cardiff University, Park Place, Cardiff CF10 3AT, Wales, UK catalytic reactions [1], present the greatest opportunities for understanding such processes at a detailed fundamental level. Among the techniques that are routinely used for physicochemical characterization of solid materials, solid-state NMR spectroscopy is a very powerful and versatile technique for investigating local structural properties and dynamic properties. However, adapting this technique for in situ studies of chemical processes can be associated with significant technical challenges. These challenges include the fact that highresolution solid-state NMR spectra are usually recorded under conditions of rapid sample spinning (at frequencies typically in the range of about $3000-15,000$ revolutions per second; see Sect. 2) and the fact that the sample is contained within a sealed and relatively small rotor (cylindrical with length ca. $1.5 \mathrm{~cm}$ and typical diameter ca. $4-7 \mathrm{~mm}$ ) within a confined and relatively inaccessible space inside the magnet of the solid-state NMR spectrometer. For these reasons, developing in situ solid-state NMR techniques has typically lagged behind the advances that have been made in other in situ experimental strategies (for example, those based on X-ray diffraction, neutron diffraction, X-ray absorption spectroscopy, other spectroscopic methods, and microscopy techniques).

This paper discusses two in situ solid-state NMR strategies that we have developed in recent years for probing the time dependence of solid-state processes, in one case for understanding the evolution of adsorption processes in microporous and mesoporous solids, and in the other case for monitoring the evolution of processes involving the formation of solid materials from solution (e.g., crystallization processes). As amplified below, each of these in situ solid-state NMR techniques has significant opportunities for applications in heterogenous catalysis. 


\section{Brief introduction to solid-state NMR}

An essential requirement underlying the viability of solidstate NMR as a technique for in situ studies of solid-state processes is that high-resolution solid-state NMR is able to identify different local environments of the species present in the system from the specific set of peak positions (i.e., the isotropic chemical shifts) in the solid-state NMR spectrum, and hence has the potential to monitor how the local environments change as a function of time during the process under investigation. In the present context, the different local environments of interest may represent different adsorption states of a molecule within a porous catalyst material, or different solid phases present during a materials formation process. Here, we mention briefly the experimental methods that are typically required for measuring high-resolution solid-state NMR spectra of powder samples, which are now applied relatively routinely on dedicated solid-state NMR spectrometers. However, we note that the specific details of the technique used and the experimental conditions required to record high-resolution solid-state NMR spectra depend on the properties of the specific NMR-active nucleus to be studied and the features of the material under investigation.

In general, the solid-state NMR spectrum recorded for a (static) powder sample is very broad and featureless, as a consequence of the fact that the NMR spectrum is influenced by several anisotropic interactions, which include chemical shift anisotropy, direct dipoledipole interactions, indirect dipole-dipole interactions, and the quadrupolar interaction (which arises for nuclei with $I \geq 1$ ). Because of the anisotropy of these interactions, the NMR resonance frequency depends (often significantly) on the orientation of a given molecule and/or a given crystallite relative to the applied magnetic field in the NMR spectrometer. As a powder sample contains, in principle, all possible orientations of molecules and/or crystallites, with different orientations giving rise to different NMR resonance frequencies, the resultant NMR spectrum typically contains very broad lines and the contributions to the NMR spectrum from structurally distinct species in the material of interest are unresolved from each other.

Fortunately, a number of ingenious experimental methods have been developed to allow isotropically averaged (i.e., high-resolution) NMR spectra to be recorded for powder samples, in which a narrow line is observed for each structurally distinguishable environment in the material for the type of nucleus of interest. These experimental methods include magic-angle sample spinning (MAS), high-power heteronuclear decoupling, and multiple-pulse techniques. In the case of MAS, the capability to spin samples at MAS frequencies up to ca. $15 \mathrm{kHz}$ is standard on most solid-state NMR instrumentation, although it is now possible to achieve MAS frequencies of over $100 \mathrm{kHz}$ using specialized apparatus. However, we note that such ultrafast MAS is attained using cylindrical rotors with very small diameter (e.g., $0.8 \mathrm{~mm}$ ), and therefore the amount of sample is necessarily very low.

More details of the background to solid-state NMR and details of the techniques that are used to measure high-resolution solid-state NMR spectra may be found in introductory texts $[2,3]$. In the remainder of this section, we give a very brief overview of two specific cases: high-resolution solid-state ${ }^{1} \mathrm{H}$ NMR and high-resolution solid-state ${ }^{13} \mathrm{C}$ NMR, as these techniques are relevant to the examples presented in Sects. 3 and 4, respectively. We note that ${ }^{1} \mathrm{H}$ and ${ }^{13} \mathrm{C}$ are both cases of spin-1/2 nuclei (i.e. $I=1 / 2$ ), but they differ significantly in natural abundance (natural isotopic abundances: ${ }^{1} \mathrm{H}>99.9 \%$; $\left.{ }^{13} \mathrm{C} 1.1 \%\right)$.

In the case of ${ }^{1} \mathrm{H}$ NMR of solids, line broadening is usually very severe as a consequence of strong ${ }^{1} \mathrm{H} \cdots{ }^{1} \mathrm{H}$ direct dipole-dipole interactions (this situation arises both because ${ }^{1} \mathrm{H}$ nuclei in solids typically have many other ${ }^{1} \mathrm{H}$ nuclei in their close proximity and because the magnitude of the magnetogyric ratio $(\gamma)$ of ${ }^{1} \mathrm{H}$ is very high). The strong ${ }^{1} \mathrm{H} \cdots{ }^{1} \mathrm{H}$ direct dipole-dipole interactions cannot readily be eliminated by MAS (except perhaps using instrumentation that allows ultra-high MAS frequencies, of the order of $100 \mathrm{kHz}$, to be attained); furthermore, standard heteronuclear decoupling techniques cannot be used to eliminate the (homonuclear) ${ }^{1} \mathrm{H} \cdots{ }^{1} \mathrm{H}$ direct dipole-dipole interactions. However, in favorable cases (e.g., if the molecules containing the ${ }^{1} \mathrm{H}$ nuclei are dynamic in the material of interest, and/or if the ${ }^{1} \mathrm{H}$ nuclei are sparsely distributed in the material), the line broadening due to the anisotropic interactions is reduced significantly, and highresolution solid-state ${ }^{1} \mathrm{H}$ NMR spectra can be recorded simply using MAS (at normal spinning frequencies) to eliminate the residual line broadening. This situation is encountered in the system discussed in Sect. 3.3.

In the case of high-resolution solid-state ${ }^{13} \mathrm{C}$ NMR of organic solids (at natural isotopic abundance), the primary contributions to line broadening arise from chemical shift anisotropy and from ${ }^{13} \mathrm{C} \cdots{ }^{1} \mathrm{H}$ direct dipole-dipole interactions. The line broadening can be reduced significantly (resulting in high-resolution solid-state ${ }^{13} \mathrm{C}$ NMR spectra) by a combination of MAS, typically at spinning frequencies of around $10 \mathrm{kHz}$, and high-power ${ }^{1} \mathrm{H}$ decoupling. This methodology has been used to record the highresolution solid-state ${ }^{13} \mathrm{C}$ NMR spectra discussed in Sect. 4.3. 


\section{In situ solid-state NMR strategy to study adsorption processes}

\section{Introduction}

Solid-state NMR is used widely in ex situ studies of heterogenous catalytic systems, typically involving the adsorption of reagents on the catalyst outside the solid-state NMR spectrometer, followed by loading the sample into the spectrometer and measuring the NMR spectrum. However, such ex situ studies have the disadvantage that significant evolution of the system (e.g., redistribution of the populations of different adsorption sites and/or a significant extent of chemical reaction) may occur in the time interval between carrying out the adsorption process and actually starting the solid-state NMR measurement, including the period of time required to transfer the sample to the NMR spectrometer.

To overcome this problem, it would clearly be advantageous to carry out in situ solid-state NMR studies on heterogenous catalytic systems to yield complementary information to the range of other in situ experimental techniques [1, 4-7] (such as combined EXAFS/XRD, FTIR spectroscopy, and laser Raman spectroscopies) that can provide insights into the nature of active sites and mechanisms of catalytic conversion. Several strategies for in situ solid-state NMR studies of adsorption processes have been reported, notably the development of continuous-flow techniques by Hunger and coworkers [8] and the "pulsequench" or cryogenic adsorption approaches (e.g., using the CAVERN apparatus) developed by Haw and coworkers $[9,10]$. However, the design and implementation of the apparatus required for continuous-flow in situ NMR are technically demanding. Furthermore, in the "pulsequench" or cryogenic adsorption approach, the adsorption process is not actually carried out within the NMR spectrometer, so this technique does not allow the earliest stages of the adsorption process to be probed.

To circumvent challenges of this type, we have developed [11] a much simpler experimental strategy for in situ solid-state NMR studies of the evolution of adsorption processes in porous solids. The technique does not require any adaptation or modification of the NMR instrumentation and allows NMR spectra to be recorded immediately from the start of the adsorption process. The technique has been applied to reveal details of the evolution of the adsorption in a wide range of systems, including adsorption of water in nanoporous silica (MCM-41) by in situ solid-state ${ }^{1} \mathrm{H}$ NMR [12], hydration of the solid acid catalyst ZSM-5 in its ammonium form [13] including the study of preferential clustering of water molecules during hydration of this system [14], and the evolution of water clusters during hydration of H-ZSM-5 [15].

\section{Description of the technique}

The main motivation underlying the development of our in situ solid-state NMR technique [11] was to devise a procedure to allow adsorption processes to be initiated directly inside the solid-state NMR spectrometer, thus ensuring that NMR spectra can be recorded from the time at which the adsorption process commences. With this strategy, the very earliest stages of the adsorption process can be probed, and subsequent changes in the populations of different adsorption sites are monitored by repeated measurement of the solid-state NMR spectrum as a function of time.

A physical consequence of the requirement for fast sample spinning (i.e., MAS) in recording high-resolution solid-state NMR spectra is that a significant centrifugal force is imposed on the sample. In our in situ solid-state NMR strategy for studying adsorption processes, this centrifugal force is utilized as a way to "trigger" the in situ adsorption of liquid reagents within porous materials, focusing on materials of interest within the field of heterogenous catalysis. The strategy is summarized in Fig. 1.

Initially, a solid powder of the porous material is packed into the solid-state NMR rotor at relatively low packing density, and the rotor is then spun (typically at $3 \mathrm{kHz}$ ) for a short time (typically a few mins). Spinning compresses the powder sample onto the inner walls of the rotor, leading to increased packing density in this region of the rotor and an empty region at the center of the rotor (i.e., a cylindrical void running the full length of the rotor). The rotor is then removed from the NMR spectrometer, and a thin-walled glass capillary tube containing the liquid reagent to be adsorbed into the porous solid is inserted into the cylindrical void within the rotor. The rotor is then reinserted into the NMR spectrometer (without MAS at this stage), and the temperature is adjusted to the specific temperature at which

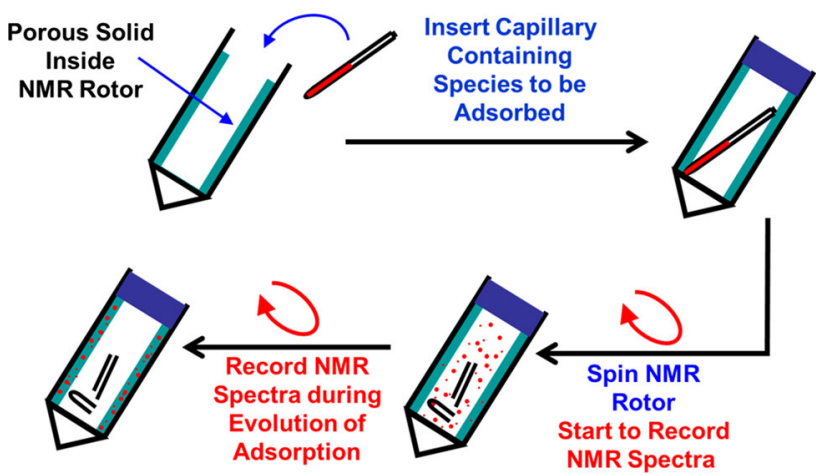

Fig. 1 Schematic illustration of the in situ solid-state NMR strategy for studying adsorption processes in porous solids 
the adsorption process is to be carried out. The rotor is then subjected to fast MAS while commencing the measurement of the solid-state NMR spectrum. Importantly, the fast sample spinning leads to breakage of the capillary tube, releasing the liquid adsorbate within the rotor (in our experience, the capillary tube breaks immediately on subjecting the rotor to MAS). As the adsorption process is triggered by bringing the adsorbate and adsorbent directly into contact within the NMR rotor, while at the same time recording the solid-state NMR data, the technique allows the very earliest stages of the adsorption process to be observed and allows the subsequent evolution of the adsorption process to be monitored as a function of time.

Clearly, the specific type of NMR measurement selected for the in situ experiment depends on the particular system under investigation and the specific aspects of the adsorption process that are to be probed. The time resolution in the in situ study is dictated by the time required to record a single NMR spectrum of adequate quality to allow meaningful information to be deduced concerning the adsorption states that exist as a function of time during the evolution of the adsorption process. Clearly, an important experimental consideration is to set up the in situ experiment such that the NMR spectra can be recorded sufficiently quickly that the time resolution of the measurement is compatible with the timescale of the changes that occur in the adsorption process.

\section{Illustrative example: probing the evolution of water clusters during hydration of the solid acid catalyst H-ZSM-5}

To illustrate the application of our technique, we focus on in situ solid-state ${ }^{1} \mathrm{H}$ NMR studies [15] of adsorption of water inside the microporous solid acid catalyst H-ZSM-5. In this work, the adsorption process was carried out at $183 \mathrm{~K}$; as water is a solid at this temperature, adsorption of water within the H-ZSM-5 sample proceeds through the vapor phase (i.e., via the sublimation of ice). After commencing sample spinning, and thus triggering the adsorption process, the high-resolution solid-state ${ }^{1} \mathrm{H}$ NMR spectrum was recorded immediately, and ${ }^{1} \mathrm{H}$ NMR spectra were then recorded repeatedly throughout the duration of the experiment. The time resolution of the in situ study (i.e., the time to record an individual ${ }^{1} \mathrm{H}$ NMR spectrum) was ca. $10 \mathrm{~min}$.

Dehydrated H-ZSM-5 has ${ }^{1} \mathrm{H}$ NMR signals at $4.3 \mathrm{ppm}$ and $1.7 \mathrm{ppm}$, assigned to Brønsted acid sites and silanol $(\mathrm{SiOH})$ groups, respectively [16]. A broad signal at ca. $7 \mathrm{ppm}$ may also be observed, and is assigned to another type of bridging hydroxyl group which interacts with other oxygen atoms of the H-ZSM-5 framework [16-18]; we refer to these bridging hydroxyl groups as "framework-
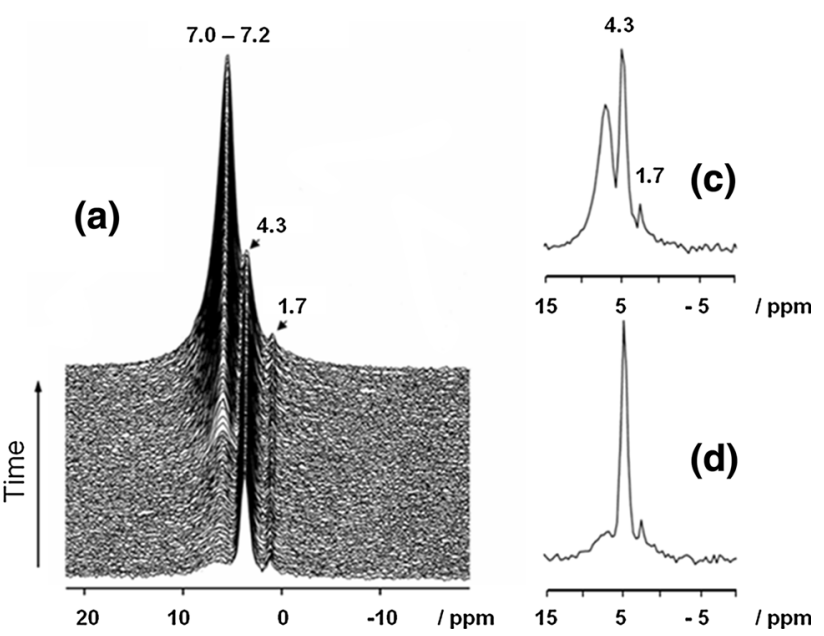

Fig. 2 a Stack plot showing all ${ }^{1}$ H MAS NMR spectra recorded as a function of time during the in situ study of adsorption of water in H-ZSM-5 at $183 \mathrm{~K}$ (time resolution, $640 \mathrm{~s}$ per spectrum; total time, $59,200 \mathrm{~s})$. The following individual ${ }^{1} \mathrm{H}$ MAS NMR spectra are also shown: b the first spectrum recorded (representing dehydrated $\mathrm{H}-\mathrm{ZSM}-5$ ), and $\mathbf{c}$ the spectrum recorded at $8.3 \mathrm{~h}$. More details of the experimental conditions are given in Ref. [15]

interacting" bridging hydroxyl groups (as distinct from the "free" Brønsted acid sites that give rise to the peak at $4.3 \mathrm{ppm})$.

In our in situ ${ }^{1} \mathrm{H}$ NMR study, the first ${ }^{1} \mathrm{H}$ NMR spectrum (Fig. 2a, b) only contains peaks at 1.7 ppm, 4.3 ppm, and ca. 7 ppm, characteristic of dehydrated H-ZSM-5. As time progresses, the intensity of the peak due to "free" Brønsted acid sites ( $4.3 \mathrm{ppm})$ decreases, and concurrently, a new peak emerges at $7.2 \mathrm{ppm}$ and grows in intensity (Fig. 2a, c). The new peak at $7.2 \mathrm{ppm}$ overlaps the broad peak at ca. $7 \mathrm{ppm}$ (assigned to "framework-interacting" bridging hydroxyl groups in the original sample of dehydrated H-ZSM-5).

The new peak at $7.2 \mathrm{ppm}$ differs from that for physically adsorbed water molecules in other silica-based materials [e.g., the siliceous nanoporous material MCM-41 (3.8 ppm [12]) or silica gel (3.5 ppm [19])] and differs from that for water molecules interacting with silanol groups $(5.4 \mathrm{ppm}$ [12]). In contrast, the significantly higher chemical shift of the peak at $7.2 \mathrm{ppm}$ is consistent with water molecules interacting with Brønsted acid sites [19]. Although the water molecules interacting with a given Brønsted acid site may encompass a range of structurally distinguishable environments, individual ${ }^{1} \mathrm{H}$ resonances for distinct water environments are not resolved in the present case, both because the peaks in the ${ }^{1} \mathrm{H}$ NMR spectrum may retain some broadening due to incompletely averaged ${ }^{1} \mathrm{H} \cdots{ }^{1} \mathrm{H}$ direct dipole-dipole interactions and because exchange of water molecules is likely to occur among different water environments at the Brønsted acid site. As a consequence, a single (averaged) ${ }^{1} \mathrm{H}$ NMR peak is observed for all the 
water molecules interacting with the Brønsted acid site. The peak at $7.2 \mathrm{ppm}$ shifts only slightly (to lower chemical shift) as the amount of adsorbed water increases.

For the original sample of dehydrated H-ZSM-5, the peak at 4.3 ppm represents "free" Brønsted acid sites that do not interact with water molecules. The intensity of this peak decreases with time during the adsorption process, indicating that the ${ }^{1} \mathrm{H}$ NMR peak for Brønsted acid sites that interact with water molecules (i.e., "hydrated" Brønsted acid sites) is shifted from $4.3 \mathrm{ppm}$. As the only peak that increases in intensity during the adsorption process is the new peak at $7.2 \mathrm{ppm}$ (already assigned to water molecules), it is clear that the hydrated Brønsted acid sites must also contribute to this peak. Thus, the broad peak at $7.2 \mathrm{ppm}$ arises from the adsorbed water molecules interacting with Brønsted acid sites, the hydrated Brønsted acid sites, and the "framework-interacting" bridging hydroxyl groups (at ca. 7 ppm). The ${ }^{1} \mathrm{H}$ chemical shift (1.7 ppm) and

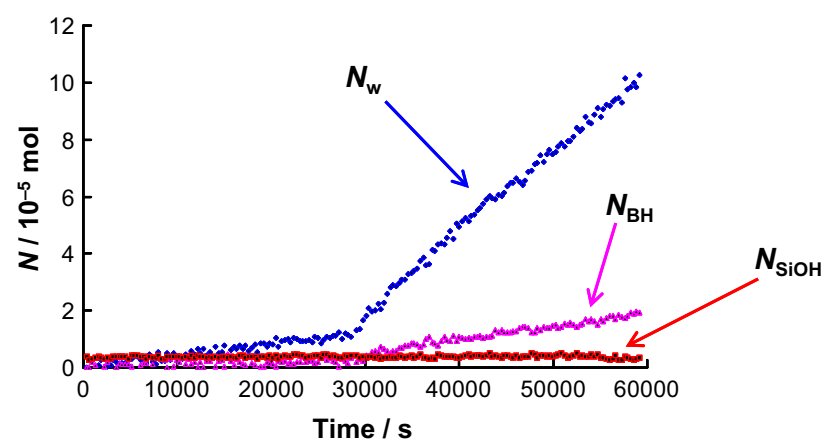

Fig. 3 Results from quantitative lineshape fitting of the in situ ${ }^{1} \mathrm{H}$ MAS NMR spectra recorded as a function of time at $183 \mathrm{~K}$ during the adsorption of water on H-ZSM-5. Blue diamonds represent the total amount of water adsorbed $\left(N_{\mathrm{w}}\right)$. Pink triangles represent the total amount of Brønsted acid sites that interact with water molecules $\left(N_{\mathrm{BH}}\right)$. Red squares represent the total amount of silanol groups $\left(N_{\mathrm{SiOH}}\right)$

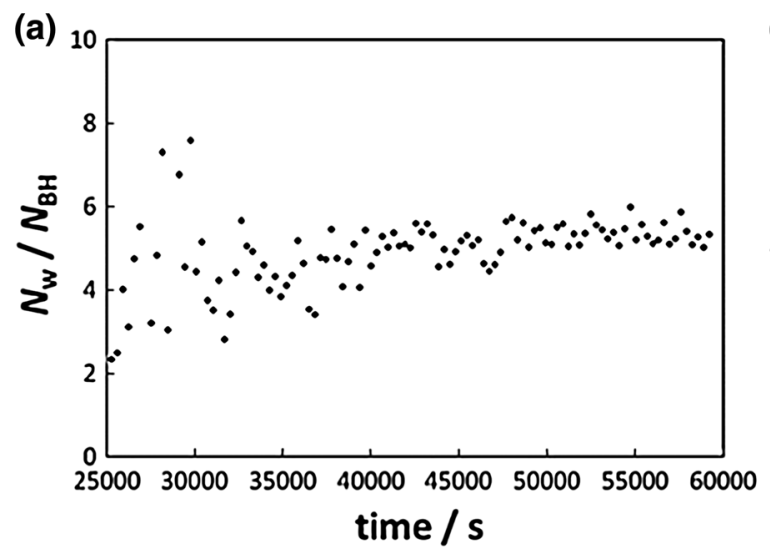

Fig. 4 a The ratio $N_{\mathrm{w}} / N_{\mathrm{BH}}$ (the average number of water molecules per hydrated Brønsted acid site) as a function of time. b The ratio $N_{\mathrm{w}} /$ $N_{\text {BH }}$ plotted versus $N_{\mathrm{BH}} / N_{\mathrm{B}}^{\text {total }}$ (the fraction of Brønsted acid sites that intensity of the peak for the silanol groups remain constant throughout the adsorption process (Figs. 2, 3).

More detailed insights on the adsorption process may be established from the time dependence of the number of water molecules that interact with Brønsted acid sites (denoted $N_{\mathrm{w}}$ ) and the time dependence of the number of hydrated Brønsted acid sites (denoted $N_{\mathrm{BH}}$ ), which can be established by appropriate integration of the in situ ${ }^{1} \mathrm{H}$ NMR data (see Appendix in Ref. [15]).

Plots of $N_{\mathrm{w}}(t)$ versus time $(t)$ and $N_{\mathrm{BH}}(t)$ versus time ( $t$ ) are shown in Fig. 3, and plots of $N_{\mathrm{w}}(t) / N_{\mathrm{BH}}(t)$ versus time $(t)$ and $N_{\mathrm{w}}(t) / N_{\mathrm{BH}}(t)$ versus $N_{\mathrm{BH}}(t) / N_{\mathrm{B}}^{\text {total }}$ are shown in Fig. 4 (where $N_{\mathrm{B}}^{\text {total }}$ is the total number of Brønsted acid sites). Importantly, $N_{\mathrm{w}} / N_{\mathrm{BH}}$ represents the average number of water molecules that interact with each hydrated Brønsted acid site, and $N_{\mathrm{BH}} / N_{\mathrm{B}}^{\text {total }}$ represents the fraction of Brønsted acid sites that are hydrated. At early stages of the adsorption process, when $N_{\mathrm{w}}$ and $N_{\mathrm{BH}}$ are both low (e.g. for $t<30,000 \mathrm{~s}$ in Fig. 3), $N_{\mathrm{w}} / N_{\mathrm{BH}}$ exhibits large statistical fluctuations; for this reason, Figs. 3 and 4 focus on the period of the adsorption process for $t>25,000 \mathrm{~s}$.

As shown in Fig. 3, both $N_{\mathrm{w}}$ and $N_{\mathrm{BH}}$ increase linearly with time. At a time $(t)$ around $29,000 \mathrm{~s}$ after starting the adsorption process, there are abrupt changes in the rate of increase of $N_{\mathrm{w}}$ and in the rate of increase of $N_{\mathrm{BH}}$. The abrupt change in the rate of adsorption at $t \approx 29,000 \mathrm{~s}$ defines two regimes of the hydration process, and we focus here primarily in the second regime, during which the major extent of water adsorption takes place. At the time of the abrupt change in the rate of adsorption, the fraction of Brønsted acid sites that are hydrated (i.e., $N_{\mathrm{BH}} / N_{\mathrm{B}}^{\text {total }}$ ) is $c a$. 0.17 , while at the end of the experiment $(t=59,200 \mathrm{~s})$, the fraction of Brønsted acid sites that are hydrated is $N_{\mathrm{BH}} /$ $\mathrm{N}_{\mathrm{B}}^{\text {total }} \approx 0.49$.

The ratio $N_{\mathrm{w}} / N_{\mathrm{BH}}$ represents the average number of water molecules associated with each hydrated Brønsted

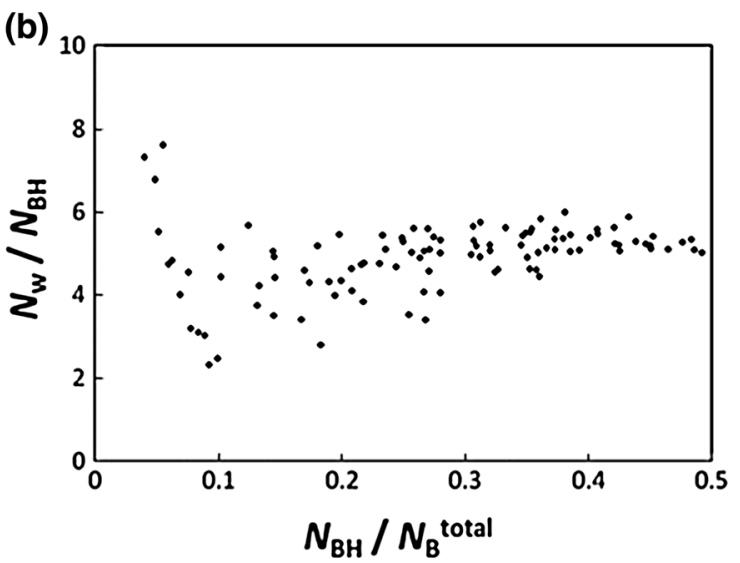

are hydrated). Data are shown for the time period $t=25,000-59,200 \mathrm{~s}$ in the in situ ${ }^{1} \mathrm{H}$ MAS NMR spectra recorded at $183 \mathrm{~K}$ 
acid site, and the time dependence of this ratio is shown in Fig. 4a. As discussed above, values of $N_{\mathrm{w}} / N_{\mathrm{BH}}$ exhibit significant statistical scatter at low values of time, but from $t \approx 25,000 \mathrm{~s}$ and throughout the remainder of the adsorption process, the value of $N_{\mathrm{w}} / N_{\mathrm{BH}}$ converges on a constant value in the range $N_{\mathrm{w}} / N_{\mathrm{BH}} \approx 5-6$ (Fig. 4). At the end of the experiment, the average size of water clusters per Brønsted acid site (averaged over the time from $50,000 \mathrm{~s}$ to $59,200 \mathrm{~s}$ ) is $N_{\mathrm{w}} / N_{\mathrm{BH}}=5.4$.

At the end of the experiment, $49 \%$ of the Brønsted acid sites are hydrated. The fact that the average number of water molecules associated with each hydrated Brønsted acid site is as high as 5.4, while $51 \%$ of Brønsted acid sites remain "free" (i.e., non-hydrated), indicates that the distribution of water molecules among the Brønsted acid sites is distinctly non-uniform. As shown in Fig. 4, the same distribution comprising both non-hydrated Brønsted acid sites and hydrated Brønsted acid sites with an average of 5-6 water molecules per hydrated site persists throughout the hydration process (at least during the second regime for $t>29,000 \mathrm{~s}$ ), and only the relative amounts of hydrated and non-hydrated Brønsted acid sites change with time. These observations imply an adsorption model in which there is a significant preference to form clusters of water molecules of a specific preferred size interacting with the Brønsted acid sites (see Fig. 5a, c), rather than a model in which all Brønsted acid sites are hydrated uniformly (see Fig. 5b, d). Although $N_{\mathrm{w}} / N_{\mathrm{BH}}$ represents an average number of water molecules associated with each hydrated Brønsted acid site, and does not provide information on the actual distribution of sizes of water clusters, the time evolution of $N_{\mathrm{w}} / N_{\mathrm{BH}}$ provides significant insights on mechanistic aspects of the adsorption process, and serves as basis to rule out a range of alternative hydration models.

For example, for a hydration model (Fig. 5b) in which all Brønsted acid sites are hydrated uniformly throughout the adsorption process (initially involving hydration of all Brønsted acid sites by a single water molecule, followed by hydration of all Brønsted acid sites by a second water molecule, and so on), the value of $N_{\mathrm{w}} / N_{\mathrm{BH}}$ would remain constant at $N_{\mathrm{w}} / N_{\mathrm{BH}}=1$ up to the point at which the fraction of hydrated Brønsted acid sites reaches $N_{\mathrm{BH}} / N_{\mathrm{B}}^{\text {total }}=1$, and should then increase linearly with further adsorption of water (see Fig. 5d). Clearly, the actual time evolution of $N_{\mathrm{w}} / N_{\mathrm{BH}}$ for hydration of H-ZSM-5 observed from our in situ solid-state ${ }^{1} \mathrm{H}$ NMR data (Fig. 4a) is different from the behavior predicted for this model, but instead is fully consistent with the model (Fig. 5a) in which the formation of clusters of a specific preferred size (ca. 5-6 water molecules) is formed at each hydrated Brønsted acid site (see Fig. 5c). (a)

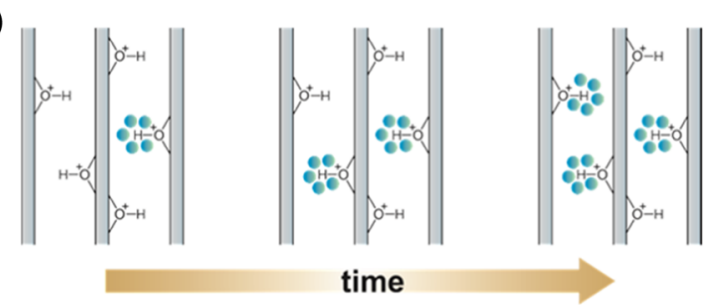

(b)

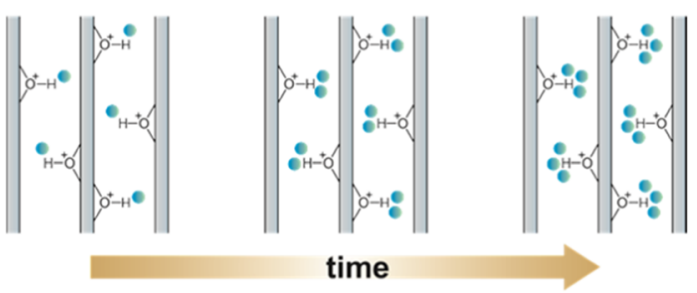

(c)

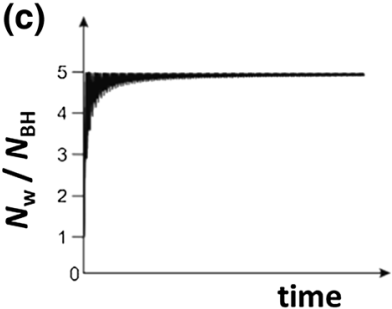

(d)

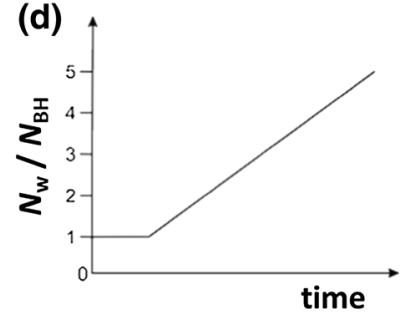

Fig. 5 Schematic illustrations of two different models for adsorption of water molecules (represented as blue circles) inside the pores of a zeolitic solid, in which the adsorbed water molecules interact specifically with the Brønsted acid sites of the zeolite (OH groups shown on the framework). a A model involving preferential formation of clusters comprising five water molecules at the Brønsted acid sites; during the hydration process, the Brønsted acid sites either interact with the preferred cluster of five water molecules or do not interact with any water molecules. b A model involving uniform hydration of all Brønsted acid sites, in which all Brønsted acid sites interact initially with one water molecule, then two water molecules, and so on, as the hydration process progresses. The time dependence of the number of water molecules per hydrated Brønsted acid site (i.e., $N_{\mathrm{w}} /$ $N_{\mathrm{BH}}$ ) during the adsorption process (assuming a constant rate of adsorption of water molecules) is shown: $\mathbf{c}$ for the model involving preferential formation of water clusters at the Brønsted acid sites, and d for the model involving uniform hydration of Brønsted acid sites. As shown in Fig. 4a, our experimental results for adsorption of water in H-ZSM-5 are consistent with the model involving preferential formation of water clusters at the Brønsted acid sites

\section{In situ solid-state NMR strategy to study materials formation processes from solution}

\section{Introduction}

Materials formation processes are encountered in many different scientific fields, and a deeper understanding of such processes has important practical implications, including the optimization of industrial applications. Improving our fundamental mechanistic understanding relies on developing new experimental strategies, particularly those that allow direct in situ monitoring of the 
process. In many cases, materials formation processes are governed by kinetic factors. Thus, meta-stable solid forms (e.g., meta-stable polymorphs or solvate materials) are often produced initially (rather than the thermodynamically stable form), and the process often evolves through a sequence of different transient solid forms before reaching the final product. To optimize and control such processes, it is essential to understand the sequence of events that occur in the evolution of the solid phase, rather than simply characterizing the final product recovered at the end of the process. Exploiting experimental strategies that allow direct in situ monitoring of materials formation processes is clearly essential in pursuit of this aim. For example, in the quest to produce new solid heterogeneous catalyst materials with specific targeted structural properties, understanding fundamentals of the process of materials synthesis may be vitally important, and again, in situ techniques to monitor the time evolution of the process may yield significant new insights.

In recent years, we have developed [20-25] new in situ solid-state NMR strategies for mapping the evolution of the solid phase during the process of materials formation from solution. While we have so far applied this technique primarily (although not exclusively) to study crystallization of organic materials from solution, the technique could equally be applied to study the formation of solids of direct relevance within the domain of heterogenous catalysis (for example, metal-organic-framework materials, zeolites, and aluminophosphates), provided two important criteria are satisfied: (a) the temperature of the process must be within the accessible range for solid-state NMR instrumentation (for which the typical upper limit is ca. $120^{\circ} \mathrm{C}$, except for NMR instruments with specialized laser-heating capabilities), and (b) in general, the target material and the reagents should not be paramagnetic.

While we plan to apply this technique to explore the formation processes of heterogeneous catalyst materials in the future, we focus here on highlighting the experimental methodology and presenting an illustrative example that reveals the level of detail that can be obtained on the evolution of different solid forms (and interconversion between solid forms) during crystallization of an organic system from the solution state.

Importantly, the in situ solid-state NMR technique [20] that we have developed for studying the time evolution of materials formation processes exploits the ability of NMR to selectively detect the solid phase in the types of heterogeneous solid/liquid system that exist during crystallization from solution, with the liquid phase "invisible" to the measurement. This technique has been shown to be a powerful approach for establishing the sequence of solid phases produced during crystallization [21-23] and for the discovery of new (transient) solid forms [24]. (a)

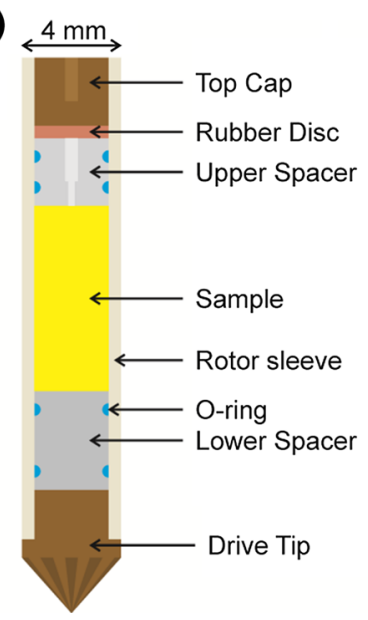

(b)

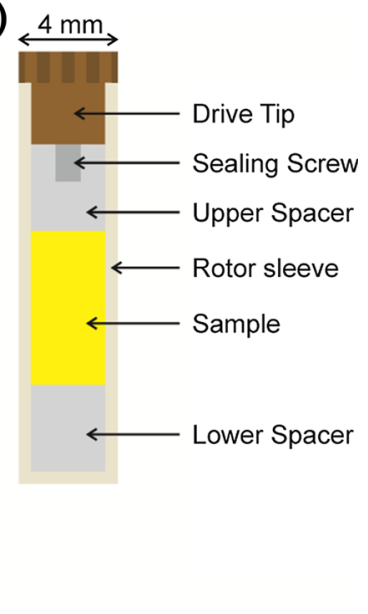

Fig. 6 Schematics of rotor designs that have been developed to overcome the challenges of sealing liquid-containing samples inside NMR rotors for rapid MAS experiments, as required for in situ solidstate NMR studies of materials formation from solution. The designs shown represent schematics of the sealing systems used in NMR rotors supplied by (a) Varian, and (b) Bruker

\section{Description of the technique}

Until recently, the prospect of using solid-state NMR for in situ studies of crystallization from solution was limited by the difficulty of securely sealing a fluid phase inside an NMR rotor, such that fast MAS (which is required for recording high-resolution solid-state NMR spectra-see Sect. 2) could be carried out without problems arising from leakage of the liquid from the rotor. Recently, suitable rotor technology has been developed for sealing solutions inside NMR rotors for MAS experiments (Fig. 6). This technical advance greatly facilitated the development of the in situ solid-state NMR strategy described here.

In our in situ solid-state NMR strategy for monitoring crystallization (see Fig. 7), a homogeneous (undersaturated) solution is initially prepared inside the NMR rotor at high temperature. Crystallization is induced by decreasing the temperature rapidly to a target temperature at which the solution is supersaturated, and hence crystallization is thermodynamically favored. The time dependence of the crystallization process is monitored by recording highresolution solid-state NMR spectra repeatedly as a function of time. The time resolution of the in situ monitoring of the crystallization is dictated by the time required to record an individual NMR spectrum of adequate quality to identify and distinguish the different solid forms present during the evolution of the system. Sufficiently good spectral resolution is also required to identify and assign the solid phases present at different stages of the crystallization process. Clearly, it is desirable to be able to detect and identify the first solid particles produced in the crystallization process, at which stage the amount of solid in the system is 

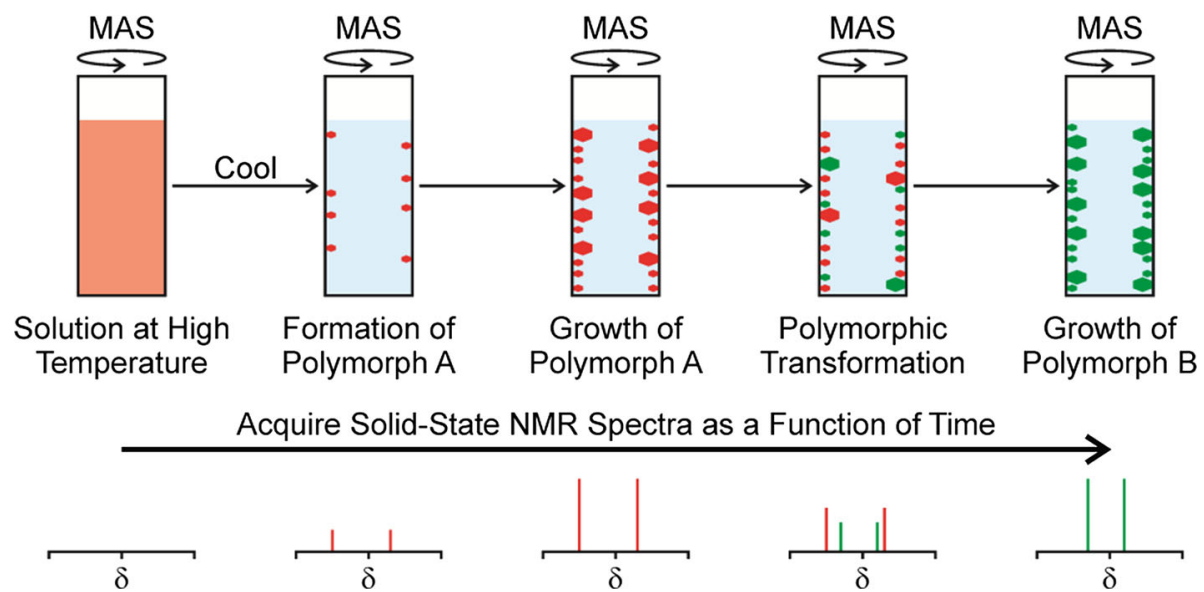

Fig. 7 Schematic of the experimental strategy for in situ solid-state NMR studies of materials formation processes, illustrated for a system in which crystallization from solution initially produces a meta-stable polymorph A (red) followed by a polymorphic transformation to produce a more stable polymorph B (green). The corresponding changes in the high-resolution solid-state NMR spectrum as a function of time are shown at the bottom generally very low. Thus, optimization of the sensitivity of the measurement is also important, allowing solid-state NMR spectra of adequate quality to be recorded in the shortest possible time. To maximize the sensitivity, isotopic labeling of the material to be crystallized is desirable (although not always essential), and carrying out the experiments at high magnetic field is advantageous.

A crucial feature of the in situ solid-state NMR strategy for monitoring crystallization is that, by appropriate choice of the measurement conditions, solid-state NMR can give complete selectivity in detecting only the solid component in the system (and thus, the dissolved solute and solvent are undetected in the measurement). For organic materials, such discrimination between solid and solution phases is readily achieved by recording ${ }^{13} \mathrm{C}$ NMR spectra using the ${ }^{1} \mathrm{H} \rightarrow{ }^{13} \mathrm{C}$ cross-polarization $(\mathrm{CP})$ technique. As a consequence of differences in dynamic behavior between molecules in the solid and solution states, ${ }^{13} \mathrm{C}$ NMR spectra recorded using ${ }^{1} \mathrm{H} \rightarrow{ }^{13} \mathrm{C} \mathrm{CP}$ contain signals only from the solid phase. Thus, even if only a small fraction of the solute has crystallized (e.g., in the early stages of crystallization), only the solid particles contribute to the measured NMR spectrum, and the dissolved solute (present in much higher amount in the early stages of crystallization) and solvent are "invisible" to the measurement.

Our most recent development of the in situ NMR technique [25], which has the potential to yield significantly deeper insights into crystallization processes than the version described above, exploits the fact that NMR spectroscopy is able to study both the liquid phase and the solid phase in a heterogeneous solid/liquid system using the same instrument, simply by changing the pulse sequence used to record the NMR data. Specifically, by alternating between two different pulse sequences in an in situ NMR study of crystallization, alternate solid-state NMR and liquid-state NMR spectra are recorded, yielding essentially simultaneous information on the time evolution of both the solid phase and the liquid phase during the crystallization process. This strategy is called "CLASSIC NMR" (Combined Liquid- And Solid-State In situ Crystallization NMR). The CLASSIC NMR experiment creates the opportunity to elucidate the complementary changes that occur in the solid phase and in the liquid phase as a function of time during materials formation from solution. During the crystallization process, the changes in the amount and the identity of the solid phase are established from the time evolution of the solid-state NMR spectrum. Concomitantly, the solution phase becomes more dilute as the solid material is formed, and changes in the solutionstate speciation and modes of molecular aggregation in solution are monitored from the time evolution of the liquid-state NMR spectrum.

\section{Illustrative example: evolution of different solid forms in crystallization of glycine from solution}

To illustrate the application of the in situ solid-state NMR technique, we focus on studies of crystallization of glycine $\left(\mathrm{H}_{3}{ }^{+} \mathrm{NCH}_{2} \mathrm{CO}_{2}{ }^{-}\right)$from different solvents. Under ambient conditions, three polymorphs of glycine (denoted $\alpha, \beta$, and $\gamma)$ are known [26-30]. The thermodynamically stable form is the $\gamma$ polymorph, while the least stable form is the $\beta$ polymorph [31, 32]. According to the literature, crystallization of glycine from water at neutral $\mathrm{pH}$ produces the meta-stable $\alpha$ polymorph. However, a paper published in 1961 suggested [28] that crystallization of glycine from deuterated water may promote the formation of the $\gamma$ polymorph, although systematic studies of this isotope 
effect were only reported recently [33, 34], in which it was demonstrated inter alia that deuteration (even as low as $1 \%$ deuteration) does significantly increase the probability of obtaining the $\gamma$ polymorph. In high-resolution solid-state ${ }^{13} \mathrm{C}$ NMR spectra, the isotropic ${ }^{13} \mathrm{C}$ chemical shifts for the carboxylate carbon environment in the $\alpha, \beta$, and $\gamma$ polymorphs of glycine are 176.5, 175.5, and $174.5 \mathrm{ppm}$ respectively [35], allowing the three polymorphs to be readily distinguished.

In the in situ ${ }^{13} \mathrm{C}$ NMR study of crystallization of glycine (for a sample ${ }^{13} \mathrm{C}$-labeled in both carbon sites in the molecule) [21] from water with natural isotopic abundance (Fig. 8a), a peak emerges at $176.5 \mathrm{ppm}$, and the intensity increases as a function of time. From the ${ }^{13} \mathrm{C}$ chemical shift, this solid phase is assigned as the $\alpha$ polymorph. Thus, the formation and growth of the $\alpha$ polymorph are observed, consistent with the literature, and no detectable amounts of the $\beta$ or $\gamma$ polymorphs are observed during the crystallization experiment (which was carried out for a total time of $13 \mathrm{~h}$ ).

To explore the isotope effect discussed above, crystallization of glycine (for a sample ${ }^{13} \mathrm{C}$-labeled in both carbon sites in the molecule) from deuterated water was studied. The total level of deuteration of all exchangeable hydrogen sites in the system (i.e., water molecules and $\mathrm{NH}_{3}{ }^{+}$groups of the zwitterionic glycine molecules) was $86 \%$. From the in situ solid-state ${ }^{13} \mathrm{C}$ NMR results (Fig. 8b), it is clear that the $\alpha$ polymorph is again the first solid form produced in the crystallization process, suggesting that the same nucleation pathway is followed in both $\mathrm{H}_{2} \mathrm{O}$ and $\mathrm{D}_{2} \mathrm{O}$. The amount of the $\alpha$ polymorph increases during the first $1.5 \mathrm{~h}$ of the crystallization process. However, at this time, a new peak emerges at $174.5 \mathrm{ppm}$, characteristic of the $\gamma$ polymorph. The intensity of this new peak then increases with time, while the intensity of the peak due to the $\alpha$ polymorph decreases. The relative amounts of the $\alpha$ and $\gamma$ polymorphs present as a function of time, established from integrated peak intensities (corrected to allow for the different cross-polarization efficiencies of the $\alpha$ and $\gamma$ polymorphs), are shown in Fig. 8c. There is no evidence for any intermediate solid phase in the transformation from the $\alpha$ polymorph to the $\gamma$ polymorph, consistent with the rate of increase of the $\gamma$ polymorph mirroring the rate of decrease of the $\alpha$ polymorph (Fig. 8c). For each of the two isotopomeric systems, the final polymorph obtained at the end of the in situ solid-state NMR study (i.e., the $\alpha$ polymorph in the natural abundance system and the $\gamma$ polymorph in the deuterated system) is consistent with the preferred polymorphic outcome observed in conventional (ex situ) laboratory crystallization experiments [34] carried out under the same conditions and over the same total period of time.

The results from an in situ solid-state ${ }^{13} \mathrm{C}$ NMR study of crystallization of glycine from methanol/water [22] are shown in Fig. 9 (in this experiment, the glycine sample was ${ }^{13} \mathrm{C}$-labeled only in the carboxylate group, giving significantly narrower peaks than those in Fig. 8a, b). In the first spectrum recorded, the solid phase is identified as a virtually pure sample of the $\beta$ polymorph (with a very small

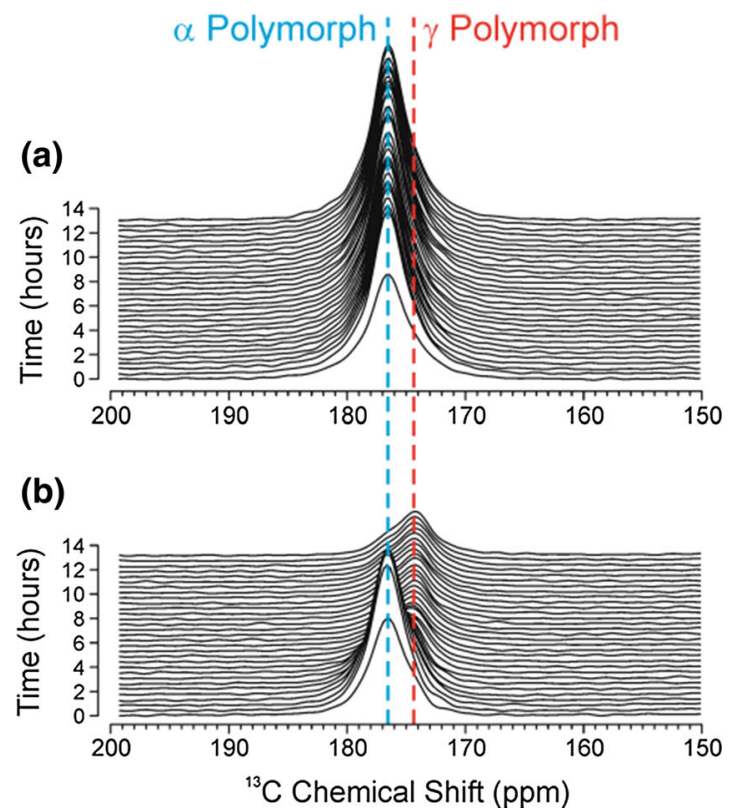

Fig. 8 In situ solid-state ${ }^{13} \mathrm{C}$ NMR spectra (showing only the carboxylate region) recorded as a function of time during crystallization of glycine from (a) $\mathrm{H}_{2} \mathrm{O}$ and (b) $\mathrm{D}_{2} \mathrm{O}$. (c) Relative amounts of the $\alpha$ (blue) and $\gamma$ (red) polymorphs of glycine as a function of time (c)

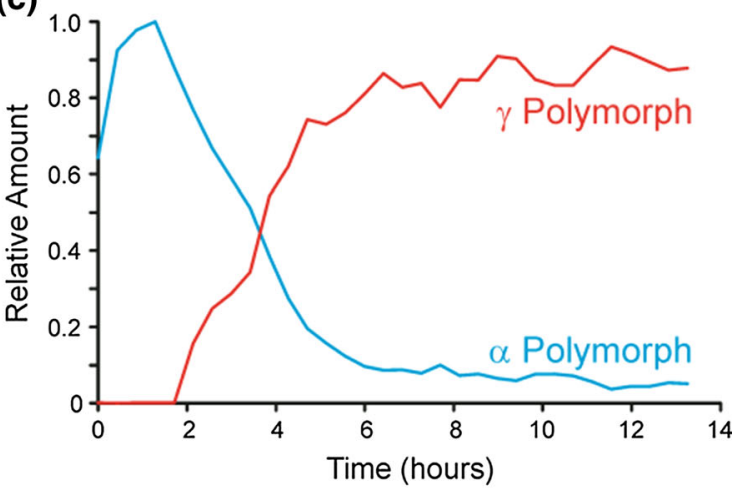

during crystallization of glycine from $\mathrm{D}_{2} \mathrm{O}$, established from the in situ solid-state ${ }^{13} \mathrm{C}$ NMR data shown in (b). More details of the experimental conditions are given in Ref. [21] 


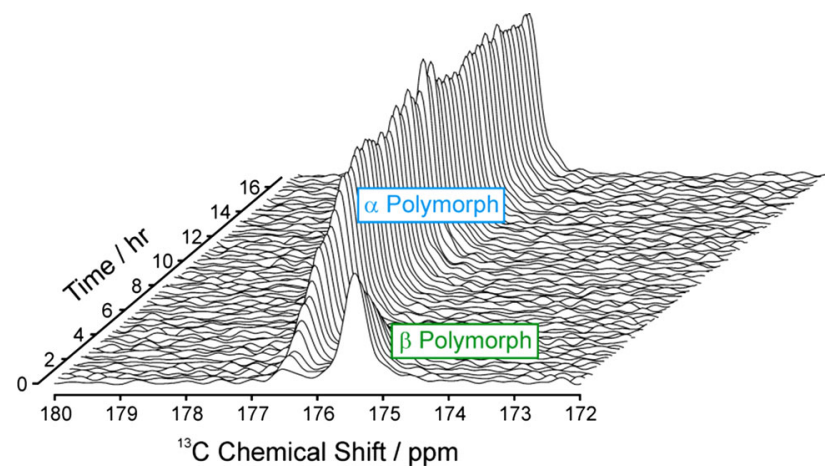

Fig. 9 In situ solid-state ${ }^{13} \mathrm{C}$ NMR spectra (showing the carboxylate region) recorded as a function of time during crystallization of glycine from methanol/water. More details of the experimental conditions are given in Ref. [22]

amount of the $\alpha$ polymorph also present). Thus, the very early stages of crystallization yield a significant excess of the $\beta$ polymorph in this system. As the crystallization process advances, the amount of the $\beta$ polymorph decreases and the amount of the $\alpha$ polymorph increases, as evident from the changes in peak intensities as a function of time in Fig. 9. The transformation from the $\beta$ polymorph to the $\alpha$ polymorph is assigned as a solution-mediated transformation. Importantly, the results from our in situ solidstate ${ }^{13} \mathrm{C}$ NMR study allow the timescale of the transformation from the $\beta$ polymorph to the $\alpha$ polymorph to be established and indicate that a viable strategy for isolating the $\beta$ polymorph would be to stop the crystallization experiment at the stage of the initial crystallization product, within only a few minutes of triggering the crystallization process.

\section{Future prospects}

The in situ solid-state NMR strategies outlined here for probing the time evolution of materials formation processes from solution, and for probing the time evolution of adsorption processes in porous solid host materials, have significant prospects for applications in areas relating to heterogenous catalysis research. Both techniques offer the potential to yield new fundamental insights that could not be gained from ex situ solid-state NMR analysis of the same systems, specifically as the in situ strategies allow the time evolution of the processes of interest to be monitored and rationalized.

In contemplating the scope and limitations of the two in situ NMR techniques discussed in this paper, a crucially important consideration is whether the experimental conditions of the NMR measurement are suitable for achieving sufficiently good time resolution in probing the adsorption process or materials formation process of interest. Clearly, adequate time resolution in in situ studies of the time evolution of these processes may be crucial to observe all adsorption states that exist during an adsorption process, or to observe all solid phases that exist during a materials formation process, including short-lived species with only transient existence. Clearly, the time resolution of the in situ study is dictated by the time required to record an individual NMR spectrum with adequate signal-to-noise ratio to allow all species that exist in the system to be identified. A number of factors are important in this regard, including: (a) the NMR receptivity of the selected nucleus, (b) the applied magnetic field strength, (c) the NMR relaxation properties, and (d) the total amount of material used in the experiment. In the case of crystallization of organic materials from solution, a more detailed discussion of these issues has been given elsewhere [23]. In general terms, the feasibility of monitoring a particular process using in situ NMR techniques depends on specific details of the process and specific details of the NMR measurement technique to be used. However, in the ideal case, the time to record an individual NMR spectrum: (a) should be shorter than the lifetime of the shortest-lived species that exists during the process (otherwise the transient existence of such short-lived species may be missed in the measurement), and (b) should be sufficiently short that many (e.g., 30-50) repeated measurements of the NMR spectrum can be completed within the overall time from the start of the process to the end of the process. Clearly, the lifetime of the transient species involved in the process and the overall time required to complete the process may be altered by varying the temperature of the experiment. In general, ensuring compatibility between the timescale of the process under investigation and the time resolution of the in situ NMR measurement technique is an important requirement for the successful application of the techniques described here, and may be achieved through a combination of optimizing the conditions of the NMR measurement technique and the experimental conditions of the process under investigation.

While our previous applications of the in situ solid-state NMR technique to study adsorption processes have been focused on materials (zeolites and mesoporous silica) and systems that are of direct relevance within the context of heterogenous catalysis, our previous studies using the in situ solid-state NMR strategy to explore materials formation processes have been focused on crystallization of organic materials from solution, with particular interest in unraveling the sequence of solid forms (e.g., polymorphs) produced during the evolution of the crystallization process. Nevertheless, there are equally exciting prospects for applying the same in situ solid-state NMR methodology to explore the time evolution of the synthesis of solid materials of relevance to applications in heterogenous catalysis. 
It is fully anticipated that the types of information revealed by this in situ NMR technique may play a pivotal role in the development of future strategies for improving, optimizing, and controlling materials synthesis processes to yield new heterogenous catalysts with specific targeted properties.

Acknowledgments I am grateful to several colleagues and collaborators who have advanced the research described in this paper, particularly Dr Colan Hughes, Dr Andrew Williams, and Dr Mingcan Xu from Cardiff University and Professor Sir John Meurig Thomas FRS from the University of Cambridge. I thank Yating Zhou for help with preparation of figures. Funding received from the KACST Oxford Petrochemical Research Centre is gratefully acknowledged. The UK High-Field $(850 \mathrm{MHz})$ Solid-State NMR Facility (located at the University of Warwick) is thanked for the award of significant amounts of time for our in situ NMR studies of crystallization processes.

Open Access This article is distributed under the terms of the Creative Commons Attribution 4.0 International License (http:// creativecommons.org/licenses/by/4.0/), which permits unrestricted use, distribution, and reproduction in any medium, provided you give appropriate credit to the original author(s) and the source, provide a link to the Creative Commons license, and indicate if changes were made.

\section{References}

1. Thomas JM (1997) The ineluctable need for in situ methods of characterising solid catalysts as a prerequisite to engineering active sites. Chem Eur J 3:1557-1562

2. Apperley DC, Harris RK, Hodgkinson P (2012) Solid state NMR: basic principles \& practice. Momentum press LLC, New York

3. Duer MJ (2004) Introduction to solid-state NMR spectroscopy. Blackwell, Oxford

4. Thomas JM (1999) Design, synthesis and in situ characterisation of new solid catalysts. Angew Chemie Int Ed 38:3589-3628

5. Sankar G, Thomas JM (1999) In situ combined X-ray absorption spectroscopic and X-ray diffractometric studies of solid catalysts. Topics Catal 8:1-21

6. Wachs IE (2005) Recent conceptual advances in the catalysis science of mixed metal oxide catalytic materials. Catal Today 100:79-94

7. Hunger M, Wang W (2006) Characterization of solid catalysts in the functioning state by nuclear magnetic resonance spectroscopy. Adv Catal 50:149-225

8. Hunger M, Horvath T (1995) A new MAS NMR probe for in-situ investigations of hydrocarbon conversion on solid catalysts under continuous flow conditions. J Chem Soc Chem Commun $14: 1423-1424$

9. Haw JF, Goguen PW, Xu T, Skloss TW, Song WG, Wang ZK (1998) In-situ NMR investigations of heterogeneous catalysis with samples prepared under standard reaction conditions. Angew Chemie Int Ed 37:948-949

10. Haw JF, Nicolas JB, Xu T, Beck LW, Ferguson DB (1996) Physical organic chemistry of solid acids: lessons from in situ NMR and theoretical chemistry. Acc Chem Res 29:259-267

11. Xu M, Harris KDM, Thomas JM, Vaughan DEW (2007) Probing the evolution of adsorption on nanoporous solids by in situ solidstate NMR spectroscopy. Chem Phys Chem 8:1311-1313
12. Xu M, Harris KDM, Thomas JM (2008) Mapping the evolution of adsorption of water in nanoporous silica by in situ solid-state ${ }^{1}$ H NMR spectroscopy. J Am Chem Soc 130:5880-5882

13. Xu M, Harris KDM, Thomas JM (2009) In situ solid-state ${ }^{1} \mathrm{H}$ NMR studies of hydration of the solid acid catalyst ZSM-5 in its ammonium form. Solid State Nucl Magn Reson 35:93-99

14. Xu M, Harris KDM, Thomas JM (2009) Preferential clustering of water molecules during hydration of the ammonium form of the solid acid catalyst ZSM-5. Catal Lett 131:16-20

15. Harris KDM, Xu M, Thomas JM (2009) Probing the evolution of water clusters during hydration of the solid acid catalyst H-ZSM5. Phil Mag 89:3001-3012

16. Hunger M (1996) Multinuclear solid-state NMR studies of acidic and non-acidic hydroxyl protons in zeolites. Solid State Nucl Magn Reson 6:1-29

17. Beck LW, White JL, Haw JF (1994) ${ }^{1} \mathrm{H}\left({ }^{27} \mathrm{Al}\right)$ double-resonance experiments in solids-an unexpected observation in the ${ }^{1} \mathrm{H}$ MAS spectrum of zeolite HZSM-5. J Am Chem Soc 116:9657-9661

18. Freude D (1995) Enhanced resolution in the ${ }^{1} \mathrm{H}$ NMR spectra of zeolite H-ZSM-5 by heteronuclear dipolar-dephasing spin-echo MAS. Chem Phys Lett 235:69-75

19. Bronnimann CE, Zeigler RC, Maciel GE (1988) Proton NMR study of dehydration of the silica gel surface. J Am Chem Soc 110:2023-2026

20. Harris KDM, Hughes CE, Williams PA (2015) Monitoring the evolution of crystallization processes by in-situ solid-state NMR spectroscopy. Solid State Nucl Magn Reson 65:107-113

21. Hughes CE, Harris KDM (2008) A technique for in situ monitoring of crystallization from solution by solid-state ${ }^{13} \mathrm{C}$ CPMAS NMR spectroscopy. J Phys Chem A 112:6808-6810

22. Hughes CE, Harris KDM (2010) Direct observation of a transient polymorph during crystallization. Chem Commun 46:4982-4984

23. Hughes CE, Williams PA, Keast VL, Charalampopoulos VG, Edwards-Gau GR, Harris KDM (2015) New in situ solid-state NMR techniques for probing the evolution of crystallization processes: pre-nucleation, nucleation and growth. Faraday Discuss 179:115-140

24. Hughes CE, Williams PA, Peskett TR, Harris KDM (2012) Exploiting in situ solid-state NMR for the discovery of new polymorphs during crystallization processes. J Phys Chem Lett 3:3176-3181

25. Hughes CE, Williams PA, Harris KDM (2014) CLASSIC NMR: an in situ NMR strategy for mapping the time-evolution of crystallization processes by combined liquid-state and solid-state measurements. Angew Chemie Int Ed 53:8939-8943

26. Albrecht G, Corey RB (1939) The crystal structure of glycine. J Am Chem Soc 61:1087-1103

27. Iitaka Y (1960) The crystal structure of beta glycine. Acta Crystallogr 13:35-45

28. Iitaka Y (1961) Crystal structure of gamma glycine. Acta Crystallogr 14:1-10

29. Jönsson P-G, Kvick $\AA$ (1972) Precision neutron diffraction structure determination of protein and nucleic-acid components III. Crystal and molecular structure of amino acid alpha glycine. Acta Crystallogr Sect B 28:1827-1833

30. Kvick А, Canning WM, Koetzle TF, Williams GJB (1980) Experimental study of the influence of temperature on a hydrogen-bonded system-crystal structure of gamma glycine at $83 \mathrm{~K}$ and $298 \mathrm{~K}$ by neutron diffraction. Acta Crystallogr Sect B 36:115-120

31. Boldyreva EV, Drebushchak VA, Drebushchak TN, Paukov IE, Kovalevskaya YA, Shutova ES (2003) Polymorphism of glycinethermodynamic aspects. Part I. Relative stability of the polymorphs. J Therm Anal Calorim 73:409-418 
32. Perlovich GL, Hansen LK, Bauer-Brandl A (2001) The polymorphism of glycine-thermochemical and structural aspects. J Therm Anal Calorim 66:699-715

33. Hughes CE, Hamad S, Harris KDM, Catlow CRA, Griffiths PC (2007) A multi-technique approach for probing the evolution of structural properties during crystallization of organic materials from solution. Faraday Discuss 136:71-89
34. Hughes CE, Harris KDM (2009) The effect of deuteration on polymorphic outcome in the crystallization of glycine from aqueous solution. New J Chem 33:713-716

35. Taylor RE (2004) Setting up ${ }^{13}$ C CP/MAS experiments. Concepts Magn Reson 22A:79-89 